\title{
TWO NEW FAINT COMMON PROPER MOTION PAIRS
}

\author{
María Teresa Ruiz ${ }^{1}$ and José Maza ${ }^{2}$ \\ Departamento de Astronomía, Universidad de Chile
}

\section{Abstract}

Spectrophotometry of the common proper motion pairs ESO439-162/163 and ESO440-55a/55b shows that the first is formed by an $\mathrm{m}_{\mathrm{v}}=18.8 \mathrm{mag}$ netic DQ white dwarf and an $\mathrm{m}_{\mathrm{v}}=19.8$ DC9 wnite dwarf separated by 23 ". ES0440-55a/55b has an $m_{v}=20.2$ red dwarf (ST=M5.1) component and a DZ7 white dwarf with $\mathrm{m}_{\mathrm{v}}=19.3$, their angular separation being $5: 4$. The proper motion of the pairs is $\mu=0.38 \pm 0.03 " / y e a r$ and $\mu=0.22 \pm 0.04 " /$ year respective1y.

\section{Observations}

As a result of a search program for faint nearby stars, done using glass copies of the ESO R Survey plates, (Ruiz et al, 1988) we have found several common proper motion pairs not previously catalogued. Figure 1 and 2 are finding charts for ESO439-162/163 and ESO440-55a/55b. Spectrophotometry of the above pairs revealed that both contained WD components. The spectra were obtained at La Silla (ESO) in March 1988 using the $3.6 \mathrm{~m}$ telescope equipped with EFOSC (Faint Objects Spectrograph and Camera) and a CCD RCA detector. With a $2 "$ slit the resolution was about $20 \AA$, the night was photometric with a seeing of $1: 5$. Three flux standards and he-Ar lamps were observed during the night for flux and wavelength calibrations. The spectrograph was rotated in order to obtain the spectra of both components of each pair at the same time. The spectra are shown in Figures $3 a, 3 b, 4 a$ and $4 b$. Integration times were 3 hours for ESO439-162/163 and 2 hours in the case of ESO440-55a/ $55 \mathrm{~b}$.

CCD photometry for ES0439-162/163 was obtained in March 1988 at Las Campanas (Carnegie) with the $1 \mathrm{~m}$ telescope and a TI chip. The results are given in Table 1 , where $B, V$ are in the Johnson system and $R$, I in the Kron-Cousins system.

1 Visiting Astronomer, European Southern Observatory (La Sil1a).

2 Visiting Astronomer, Las Campanas Observatory (Carnegie). 
TABLE 1

CCD Photometry of ESO439-162/163

\begin{tabular}{lllll}
\hline & $V$ & $B-V$ & $V-R$ & $R-I$ \\
\hline ES0439-162 & 18.77 & 0.82 & 0.53 & 0.31 \\
ES0439-163 & 19.84 & 1.14 & 0.80 & 0.13 \\
\hline
\end{tabular}

No CCD photometry is yet available for ESO440-55a/55b therefore the apparent magnitudes have been estimated from the spectra in Figures $4 a$ and $4 \mathrm{~b}$. For ESO440-55a we get an apparent visual magnitude $\mathrm{m}_{\mathrm{v}} \approx 19.3$ and for ESO440.s.jb and $\mathrm{m}_{\mathrm{v}} \approx 20.2$.

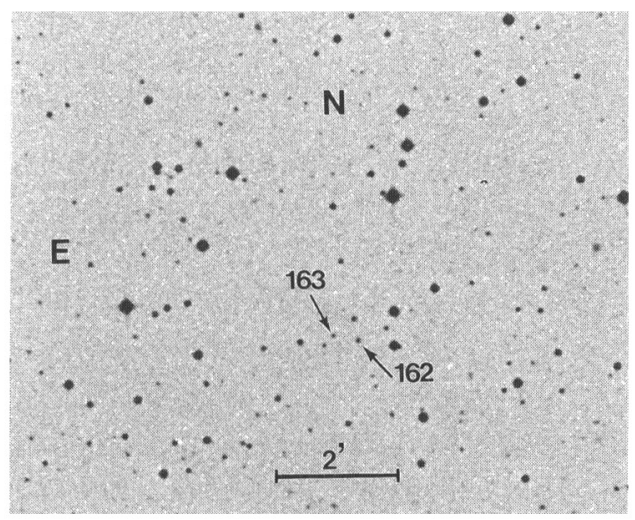

Figure 1. Finding chart for Es0439-162/163. The 1986.1 coordinates (equinox 1950.0) of ESO $439-162$ are; $\alpha=11 \mathrm{~h}_{2} 7 \mathrm{~m} 24 \mathrm{~S} 0$ and $\delta=-31^{\circ} 06^{\prime} 19^{\prime \prime}$.

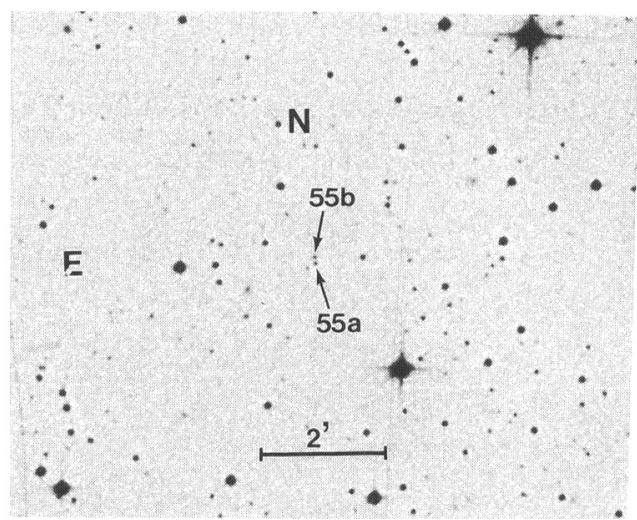

Figure 2. Finding chart for ESO $440-55 \mathrm{a} / 55 \mathrm{~b}$. The 1979.2 coordinates (equinox 1950.0) of ESO440-55a are: $\alpha=12 \mathrm{~h}_{0} 4^{\mathrm{m}_{0}} 3 \mathrm{~s}_{2}$ and $\delta=-31^{\circ} 20^{\prime} 30^{\prime \prime}$.

\section{Results}

a)ESO439-162/163: This pair formed by a magnetic DQ white dwarf (439-162) and a cold DC white dwarf (439-163) has a proper motion $\mu=0.38 \pm 0.03$ ("/year) in the direction $\theta=233^{\circ}$, the separation between the stars is 23". If we assume that the absolute visual magnitude of the cold DC star ES0439-163 is $M_{\mathrm{v}} \approx 17$ then its distance would be $35 \mathrm{pc}$, at such distance their separation would be $1.2 \times 10^{16} \mathrm{~cm}$.

The star ESO439-162 has a remarkable spectrum showing broad absorption troughs, similar only to those observed in LP790-29 whose spectral features have been identified by Liebert et al. (1978) as due to $\mathrm{C}_{2}$

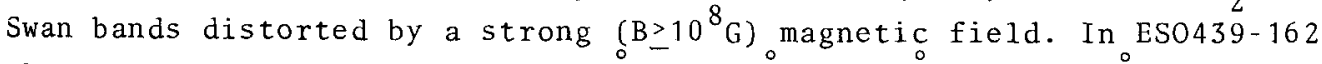
the absorptions observed at $4590 \AA, 4992 \AA, 5411 \AA$ and $5912 \AA$ correspond 


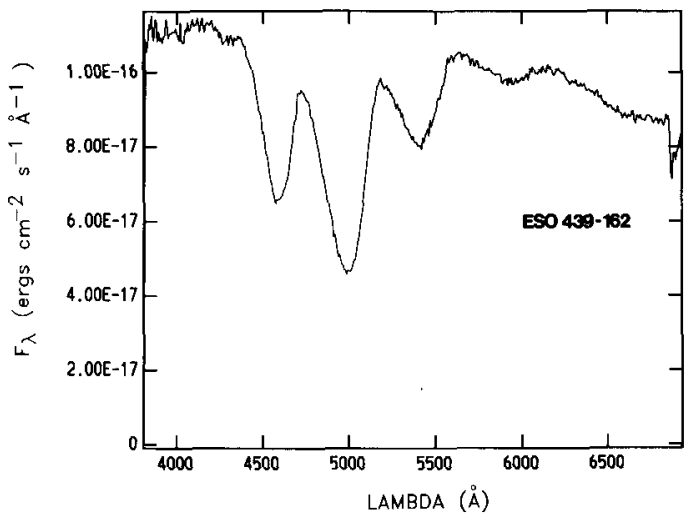

Figure 3a. Spectrum of ESO439-162. The integration time was 3 hours.

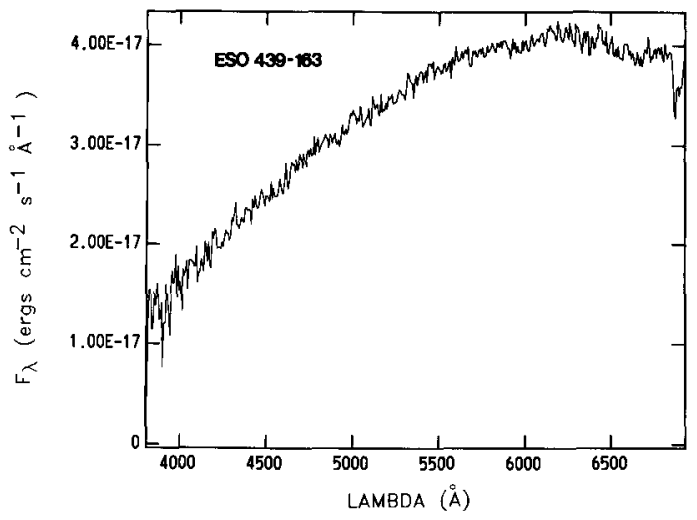

Figure 3b. Spectrum of ESO439-163 taken with an integration time of 3 hours. to those normally ( $B \approx 0)$ at $4700 \AA, 5160 \AA, 5585 \AA$ and $6100 \AA$ respectively due to the Swan band of $\mathrm{C}_{2}$. The observed displacement in wavelength of the absorptions are produced by a magnetic field $\mathrm{B} \sim 10^{8} \mathrm{G}$.

The companion star ESO439-163, has a smooth spectrum typical of DC white dwarfs with a spectral distribution that can be fitted by a $4000 \mathrm{~K}$ black body, while that of ESO439-162 corresponds (with some imagination) to a black body at $6300 \mathrm{~K}$. This difference in temperature and magnitude reflects a large difference in their cooling ages (of $10^{9}$ years or more), which would suggest that the mass of the progenitor of ES0439-162 must have been significantly less than that of its cold DC companion, contradicting the idea that the progenitors of magnetic white dwarfs are Ap stars, which have masses $>2 \mathrm{M}_{\odot}$ (Angel et a1. 1981).

b) ESO440-55a/55b: This pair, formed by a DZ7 white dwarf and an M5. 1 red dwarf, share a common proper motion $\mu=0.22 \pm 0.04$ ("/year) in the direction $\theta=273^{\circ}$. The components have an angular separation of $5: 4$.

The absolute visual magnitude of an M5.1 dwarf is $M_{v} 214.8$ (Wing and Dean, 1983) which sugggests a distance to ESO440-55b, given its $\mathrm{m}_{\mathrm{v}} \approx 20.2$, of $120 \mathrm{pc}$. 

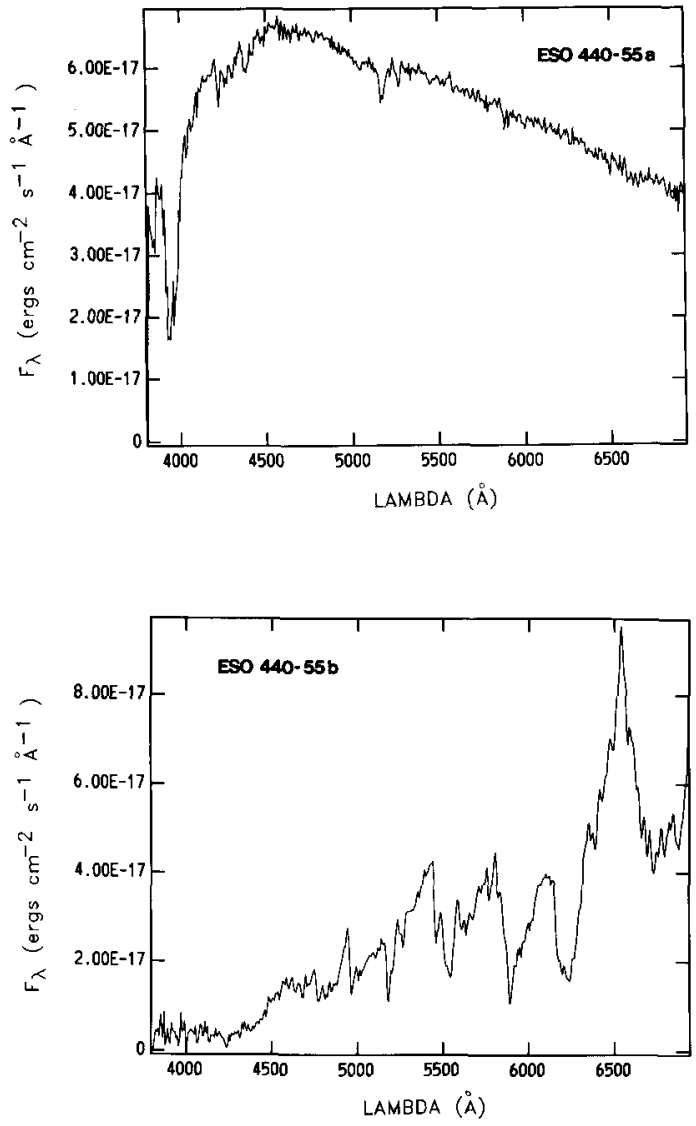

Figures $4 \mathrm{a}$ and $4 \mathrm{~b}$, Spectra of ESO440$55 \mathrm{a}$ and ESO440-55b respectively, taken with an integration time of 2 hours.
The star ESO440-55a has a DC type spectrum with lines of CaII (H and K), CaI (4227 $)$, $\operatorname{MgI}(3839 \AA, 5173 \AA+5184 \AA)$ and FeI $(4384 \AA, 5270 \AA$ and $5456 \AA$ ) no $\mathrm{H}$ or He lines are present. The spectrum can be fitted by a BB at $T \approx 7000 \mathrm{~K}$, at a distance of $120 \mathrm{pc}$ its absolute visual magnitude would be $\mathrm{M}_{\mathrm{v}} \approx 13.9$.

The mechanism most common1y invoked for the presence of metals in the atmospheres of DZ stars is that of accretion from interstellar clouds, however in order to explain the peculiar abundances in their atmospheres a selective mechanism which inhibits the accretion of $\mathrm{H}$ has to exist, up to now all of those proposed work efficiently for $T \geq 11000 \pm 1000 \mathrm{~K}$ (Liebert et a1. 1987) and for DZ stars as cold as ESO440-55a there is no satisfactory explanation for the presence of metals in their atmospheres.

We would like to thank Dr. James Liebert for very helpful comments. This research received partial support from FONDECYT grant \#359-87/88.

\section{References}

Ange 1,J.R.P., Borra,E., and Landstreet,J.D., 1981, Ap.J.Supp1., 45, 457 . Liebert,J., Angel,J.R.P., Stockman,H.S., and Beaver,E.A., 1987, Ap.J., 225, 181 .

Liebert,J., Wehrse,R., and Green,R.F., 1987, Astron. Astrophys. 175, 173. Ruiz, iM.T., Maza,J., Mendez,R., and Wischnjewsky,M., 1988, "The ESO Messenger" September issue.

Wing, R. and Dean,C.A., 1983, "IAU Colloq. N76", ed. A.G.D. Philip and A.R. Upgren, pg. 385 . 